www.jmscr.igmpublication.org

Index Copernicus Value: 79.54

ISSN (e)-2347-176x ISSN (p) 2455-0450

crossref DOI: https://dx.doi.org/10.18535/jmscr/v7i5.48

Journal Of Medical Science And Clinical Research

IGM Publication

An Official Publication of IGM Publication

\title{
Transfusion Support and Challenges in Autoimmune Hemolytic Anemia (AIHA): Experience at a Tertiary Care Centre in South India
}

Authors

\author{
Dr Parimala Puttaiah ${ }^{1}$, Dr Soumee Banerjee ${ }^{2}$, Dr Sitalakshmi Subramanian ${ }^{3}$
}

${ }^{1}$ Lecturer, Department of Transfusion Medicine and Immunohematology, St Johns Medical College ${ }^{2} 2^{\text {nd }}$ Year Postgraduate Trainee, Department of Transfusion Medicine and Immunohematology, St Johns Medical College

${ }^{3}$ Professor and Head, Department of Transfusion Medicine and Immunohematology, St Johns Medical College

Corresponding Author

Dr Sitalakshmi Subramanian

Email:slvbs@yahoo.co.in

\begin{abstract}
Background: Diagnosing patients of autoimmune hemolytic anemia (AIHA) requires an evaluation of history, clinical features and laboratory findings. In cases requiring transfusion, while serological incompatibility often makes it difficult and time-consuming to find a best match unit, it should not be an indication to withhold transfusion.

Objectives: To study the clinicopathological profile, transfusion requirements and challenges of patients of AIHA diagnosed and managed at our hospital over one year (January-December 2017).

Material and Methods: This is a descriptive retrospective study. The diagnosis of AIHA was based ondemographics and history, presenting features and hematological and biochemical findings. Tests performed-direct and indirect antiglobulin test (DAT, IAT), cold agglutinin titer (CAT); Hematological and biochemical indicators of in vivo hemolysis- hemoglobin, reticulocyte count, total serum bilirubin and serum lactate dehydrogenase (LDH). All data were obtained from patient records. Patients were divided into 2 categories-severe and moderate hemolysis. Packed red cell transfusions for these patients, including incompatible cross matches were documented.

Results: Of 185 DAT positive cases, 44 patients aged between 1 and 68years (M:F = 1:2.6) were diagnosed as AIHA with a peak incidence in the third decade. Of these, as per our criteria, moderate and severe hemolysis was shown by 29 and 15 patients respectively. Pallor was the commonest presenting feature. 19(43\%) patients had AIHA secondary to another cause, infection being the commonest. Patients showed good correlation of severity of clinical features with degree of derangement of laboratory parameters. Hb was $<8 \mathrm{gm} / \mathrm{dL}$ in both categories with 19 patients showing severe anemia $(<5 \mathrm{gm} / \mathrm{dL})$. Mean serum Bilirubin, LDH and reticulocyte count were also deranged in both categories. Concomitant IAT and CAT positivity were seen in 24 and 2 cases respectively. 179 units of PRBCs were crossmatched for these 44 cases in the study period. Of these, 84 (47\%) units were designated as "least incompatible". Ultimately 57 units were transfused to $22(50 \%$ of total) patients- 3 patients with severe hemolysis received 15 units in total(5units/patient) and 19 patients with moderate hemolysis used 42 units in total(2.2 units/patient). C:T ratio- 2.6

Conclusion: Diagnosis of AIHA is a multipronged approach. In general, degree of hemolysis, clinical and laboratory parameters and transfusion requirements correlate well. Besides DAT positivity, IAT and CAT can also be positive, making finding "best match" units in such cases difficult. Only about 50\% of patients were transfused, while the rest were managed with supplementary modalities. Serological incompatibility can adversely affect turnaround time of issue and blood utilization but should not be a reason to not transfuse.

Keywords: Autoimmune hemolytic anemia, transfusion, Direct Antiglobulin Test.
\end{abstract}




\section{Background}

Autoimmune Hemolytic Anemias (AIHA) are characterized by two key features- shortened lifespan of RBCs and presence of autoantibodies against autologous $\mathrm{RBCs}^{[1]}$. The presence of antibodies, with or without complement, coating the RBC surface is detected by a positive direct antiglobulin Test (DAT), which is essential for diagnosing a case of AIHA $^{[2]}$. However a positive DAT does not necessarily mean the patient has AIHA. Findings of the DAT must always be interpreted in conjunction with patient's history and other hematological and biochemical findings. ${ }^{[1]}$ In majority of these patients, the implicated antibodies are of $\mathrm{IgG}$ isotype. These are called warm reactive antibodies as they bind to RBCs at 37C. In others, the antibodies are cold reactive antibodies, reacting at temperatures $<37 \mathrm{C}$. These are either of cold agglutinin ( $\operatorname{IgM})$ or cold hemolysin (IgG) types ${ }^{[3]}$. In absence of any underlying disease, AIHA is termed as primary or idiopathic AIHA. AIHA can also occur secondary to causes such as underlying malignancies or autoimmune disorders or intake of certain drugs ${ }^{[4]}$. Most patients with AIHA develop anemia gradually, allowing for cardiovascular compensation and may not need any transfusions. But in those cases that do require transfusions, grouping the patient and finding a compatible unit can be quite challenging. Our study analyzes the clinical and laboratory features of patients with AIHA and their transfusion requirements.

\section{Aims and Objectives}

1. To study the clinicopathological profile of patients with AIHA

2. To study the requirements and challenges of transfusion in these patients.

\section{Materials and Methods}

This is a retrospective study done over a period of 12 months (January 2017-December 2017) at a tertiary care centre in South India. Patient demographics, clinical manifestations and histories were obtained for all cases found to be DAT positive.

These DAT positive cases were followed up with certain hematological and biochemical tests. Immunohematological tests included- an Indirect Antiglobulin Test (IAT) and a cold agglutinin Titre (CAT) wherever necessary. Other hematological parameters compiled wereHemoglobin percentage, Reticulocyte Count and Peripheral Smear findings.

Biochemical parameters studied involved- total, direct and indirect serum bilirubin, Serum Lactate Dehydrogenase (LDH).

The criteria for in-vivo hemolysis were set as defined by previous workers ${ }^{[5]}$

1. Hemoglobin $<9 \mathrm{gm} / \mathrm{dL}$,

2. Reticulocyte count- $>2 \%$

3. Serum total bilirubin- $>2 \mathrm{mg} / \mathrm{dL}$

4. Lactate dehydrogenase- $>500 \mathrm{IU} / \mathrm{mL}$

We classified patients into 2 categories to enable more effective analysis-

1. Severe Hemolysis- presence of all four laboratory features

2. Moderate Hemolysis- presence of any one, two or three laboratory features

For each case, the total number of PRBCs transfused, including the units that were serologically incompatible, were also noted. All the data were compiled from the patients' online hospital records.

All data was compiled and analysed on Microsoft Excel 2010

\section{Exclusion Criteria}

1. DAT positivity due to $\mathrm{Rh}$ and $\mathrm{ABO}$ incompatibility in newborns

2. IAT-positive in Rh-negative pregnant women

3. Patients who received blood transfusion in the previous 3 months.

\section{Results}

The total number of DAT positive cases studied during this period was 185 . Of these, 44 were diagnosed as AIHA on the basis of history, clinical features, other hematological and 
biochemical findings. The total number of males were 12 and females were 32 making the M:F ratio 1:2.6.

The ages of the patients ranged from 1-68 years with peak incidence at the $3^{\text {rd }}$ decade. Ages of patients with Moderate hemolysis ranged between 1-68years and those with severe hemolysis ranged between 9-60 years. There were 12 children below 18 years of age ( $27 \%$ of total), 7 of whom were females and 5 males. Out of the 32 adults $(73 \%), 7$ were males and 25 were females.
Of the total number of patients, moderate hemolysis was seen in 29 patients ( 8 males, 21 females) and severe hemolysis was seen in 15 patients (4 males, 11 females).

First, we looked at the features at presentation of the patients. The 3 commonest presenting features were- pallor, icterus and organomegaly. The demographics and clinical features are summarized in table 1 .

Table 1: $\%$ of major signs and symptoms seen at presentation in the cases of AIHA studied $(n=44)$

\begin{tabular}{|c|c|c|c|c|}
\hline \multirow{3}{*}{ DEMOGRAPHICS } & \multicolumn{2}{|c|}{ MODERATE HEMOLYSIS $(n=29)$} & \multicolumn{2}{|c|}{ SEVERE HEMOLYSIS(n=15) } \\
\hline & & & & \\
\hline & MALE & FEMALE & MALE & FEMALE \\
\hline Children $<18$ years $(\mathrm{n}=12)$ & 4 & 5 & 1 & 2 \\
\hline Adult $>18$ years $(\mathrm{n}=32)$ & 4 & 16 & 3 & 9 \\
\hline \multicolumn{5}{|l|}{ PRESENTING FEATURES } \\
\hline Organomegaly & \multicolumn{2}{|c|}{$9(31 \%)$} & \multicolumn{2}{|c|}{$10(66.6 \%)$} \\
\hline Icterus & \multirow{2}{*}{\multicolumn{2}{|c|}{$6(20.6 \%)$}} & \multicolumn{2}{|c|}{$11(73.3 \%)$} \\
\hline Pallor & & $29(100 \%)$ & \multicolumn{2}{|c|}{$15(100 \%)$} \\
\hline
\end{tabular}

25 cases were diagnosed as primary AIHA and in 19 cases, AIHA was secondary to other diseases like connective tissue disorders. In these 19 patients of secondary AIHA, hemolysis was moderate in 10 and severe in 9 cases. The commonest secondary causes were divided into 4 major categories, as seen in figure 1

Figure 1: Etiologies of secondary AIHA $(n=19)$ in the cases studied

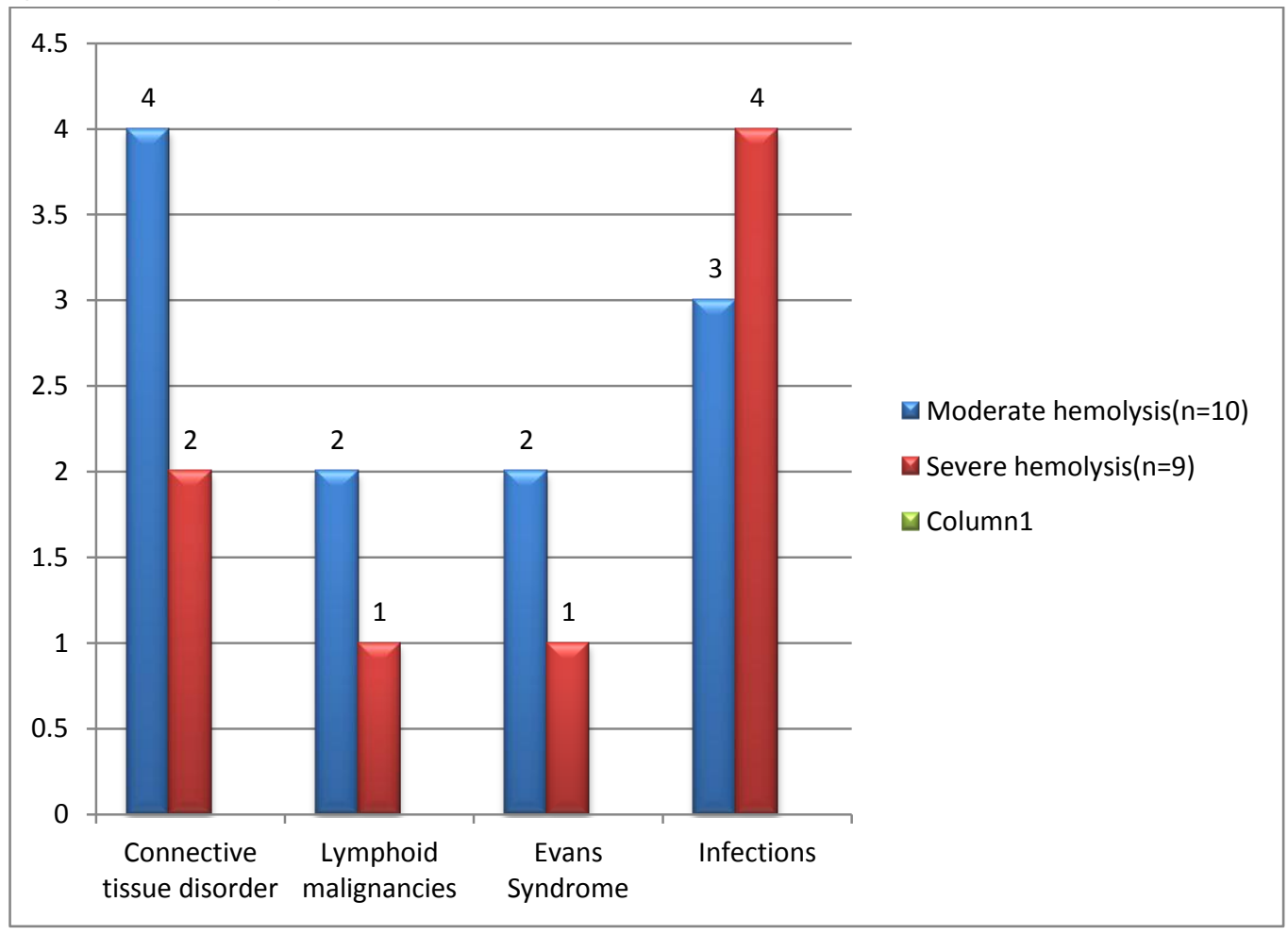


As far as the hematological and biochemical ranges from all our cases are as shown in table 2 . parameters were concerned, their mean values and

Table 2: Mean values of Hematological and biochemical parameters in the cases studied

\begin{tabular}{|c|c|c|c|c|}
\hline \multirow[t]{2}{*}{ Parameter } & \multicolumn{2}{|c|}{ Moderate hemolysis $(n=29)$} & \multicolumn{2}{|c|}{ Severe hemolysis $(n=15)$} \\
\hline & MEAN & RANGE & MEAN & RANGE \\
\hline Haemoglobin & $7.6 \mathrm{~g} / \mathrm{dl}$ & $5.6-8.7 \mathrm{gm} / \mathrm{dL}$ & $4.39 \mathrm{~g} / \mathrm{dl}$ & $3.1-5.4 \mathrm{gm} / \mathrm{dL}$ \\
\hline Reticulocyte count & $13 \%$ & $8.2-17.6 \%$ & $21 \%$ & $18.3-26.5 \%$ \\
\hline Serum bilirubin & $2.26 \mathrm{mg} / \mathrm{dl}$ & $1.2-3.3 \mathrm{mg} / \mathrm{dl}$ & $4.1 \mathrm{mg} / \mathrm{dl}$ & $3.6-5.2 \mathrm{mg} / \mathrm{dl}$ \\
\hline \multirow[t]{2}{*}{$\mathrm{LDH}$} & $536 \mathrm{IU} / \mathrm{ml}$ & 286-1066IU/mL & $1728 \mathrm{IU} / \mathrm{ml}$ & $1185-2214 \mathrm{IU} / \mathrm{mL}$ \\
\hline & \multicolumn{2}{|c|}{ \% OF TOTAL CASES } & \multicolumn{2}{|c|}{ \% OF TOTAL CASES } \\
\hline $\begin{array}{l}\text { DAT strength (3+ to } \\
4+\text { reaction) }\end{array}$ & \multicolumn{2}{|c|}{$93 \%$} & \multicolumn{2}{|c|}{$100 \%$} \\
\hline $\begin{array}{l}\text { DAT strength }(1+\text { to } \\
2+\text { reaction })\end{array}$ & \multicolumn{2}{|c|}{$7 \%$} & \multicolumn{2}{|c|}{ NIL } \\
\hline
\end{tabular}

Severe anemia $(\mathrm{Hb}<5 \mathrm{gm} / \mathrm{dL})$ was seen in 19 patients in total (severe hemolysis-14, moderate hemolysis-5). Serologically, all cases were DAT positive. Additionally, IAT was positive in $24(54.5 \%)$ cases (severe hemolysis-14, moderate hemolysis-10), CAT was significant (>1:32 dilution) in $2(4.5 \%)$ [agglutination in dilutions upto- 1:512, 1:1024 respectively] cases, both showed severe hemolysis.

When the transfusion requirements of these patients were studied, a total of 179 units of PRBCs were crossmatched for these 44 cases in the study period. Out of these, $84(47 \%)$ units were designated as "best match" or "least incompatible". Despite cross-matching, ultimately only 3 patients with severe hemolysis received 15 units in total(5units/patient) and 19 patients with moderate hemolysis used 42 units in total( 2.2 units/patient). So, 57 units were transfused to these 22 patients making C:T ratio 2.6.

\section{Discussion}

Hemolytic anemia due to immune injury can be of 4 types- warm autoantibody mediated, cold autoantibody mediated, mixed cold and warm autoantibody mediated and drug induced. ${ }^{[4]}$ While immunological tests like DAT and IAT help distinguish them from other hemolytic anemias, diagnosis entails a careful assessment of history, clinical features, hematological and biochemical parameters. $^{[6]}$ Our study sets aside clearly designated values for each of these diagnostic criteria and studies cases based on the same, over a period of one year. By those criteria, only about $23 \%$ DAT positive cases were diagnosed as AIHA. This highlights the importance of all these supplemental information in correctly assessing the implication of a positive DAT.

We found that the incidence of AIHA was much higher in females. The age range was very wide, but the peak incidence was seen in the $3^{\text {rd }}$ decade of life. As per our predefined criteria, all patients were divided into 2 categories- moderate and severe hemolysis. About $1 / 3^{\text {rd }}$ of the patients fulfilled the 4 designated criteria for hemolysis and were classified as severe hemolysis. In about $40 \%$ of the cases, AIHA was secondary to another underlying pathology, most commonly, infections and connective tissue disorders. Fatigue and pallor were the commonest symptom and sign respectively, being present in all cases. In general, the incidence of the symptoms and the signs was higher in patients who would come under the severe hemolysis category on laboratory testing. This showed good correlation between clinical and laboratory findings. The mean hemoglobin of both these categories was, however $<8 \mathrm{gm} / \mathrm{dL}$. Expectedly, the high hemolysis category showed a greater derangement in all parameters as compared to the moderate hemolysis category. Other than DAT positivity in all cases, IAT and CAT were also positive in some cases. The above findings highlight the need as well as the challenges in planning transfusion for these patients. 
Transfusion requests for these patients were analysed. While blood was requested for all cases and cross-matching performed, only about half of them actually received transfusions, showing the role of supplemental therapy like corticosteroids and the impact of cautious approach with respect to transfusions in those patients. The other half were patients who did receive transfusions showed clinical improvement despite transfusion of serologically incompatible blood. The average requirement of a patient showing severe hemolysis was about 5 units while that of a patient showing moderate hemolysis was about 2 units. While the lifespan of transfused RBCs in expected to be low in-vivo, transfusions are required in acute conditions to maintain the Oxygen supply to tissues till other modes of therapy can take effect $^{[7]}$. Our approach of dividing the cases into well-defined categories not only help establish the good correlation of clinical and laboratory features with transfusion requirements, it also highlights the difference in approach to managing cases of both categories. Of the patients who did not require transfusion, most patients presented with features of hemolysis but were hemodynamically stable at presentation. In view of their clinical presentation, most clinicians did not want to risk a serologically incompatible transfusion. They chose supplementary therapy and patients improved clinically without transfusions. However, it has been advised that in AIHA patients requiring transfusions on the basis of clinical parameters, serological incompatibility should not be a reason to withhold transfusion. Infact transfusions can be lifesaving in such cases $^{[4]}$. As seen in our study, patients receiving incompatible units also showed clinical improvement with respect to their acute presentations and were followed up with supplementary modalities once stabilized.

Also, on an average, to find one "best match" unit, more than 2 units had to be cross-matched, making the C:T ratio beyond the acceptable cutoff. This highlights how challenging it is to find suitable units for cross-match in these patients, and how this adversely affects blood utilization patterns and turn-around time of blood issue.

One limitation of our study was that we had no means to diagnose and detect DAT negative AIHA. There may have been an underrepresentation of the number of cases. Use of supplementary, more sensitive techniques may help pick up low titre antibodies that may have been missed on conventional techniques. History, clinical features, biochemical and hematological parameters can help play a very important role in deciding which cases to suspect this in ${ }^{[8]}$.

The other limitation was that monospecific DAT was not done in all cases in our study. As per our policies, we recommend all DAT positive patients to follow-up with a monospecific DAT. However, due to financial constraints of our patients, monospecific DAT is done only when requested by the treating physicians, as they deem fit, the incidence of which is quite rare. Monospecific DAT can be a valuable tool in deciding on the treatment modality of the patients ${ }^{[5]}$. As far as present study is concerned, it could have helped us with better analysis of the transfusion requirement data of the patients.

\section{Conclusion}

Diagnosis of AIHA warrants a correlation of clinical features, history, hematological, biochemical and immunohematological findings. Our study illustrated that not all DAT positive cases are cases of AIHA. In general, patients show good correlation with respect to degree of hemolysis between their clinical and laboratory parameters and transfusion requirements. While not all patients with AIHA require transfusion and can be managed with supplemental therapy, patients showing severe hemolysis may need transfusions to tide over acute crises. There are several challenges faced while deciding on transfusion for the AIHA cases that do merit transfusions. Besides DAT positivity, other immunohematological tests like IAT and CAT can also be positive, posing further challenges in testing. Finding "best match" units in such cases 
can be difficult and can adversely affect turnaround time of issue and blood utilization. However the decision to transfusion is based on clinical findings and transfusion should not be withheld in view of serological incompatibility.

\section{References}

1. Gehrs BC, Freidberg RC. Autoimmune hemolytic anemia. Am J Hematol 2002; 69:258-271.

2. Garratty G. Autoimmune hemolytic anemia. In: Garratty G, editor. Immunobiology of transfusion medicine, $1^{\text {st }}$ Ed. New York: Marcel Dekker; 1993. P 493-522

3. Reardon JE, Marques MB. Laboratory evaluation and transfusion support of patients with autoimmune hemolytic anemia. Am J Clin Pathol 2006; 125 (Suppl 1): S71-77.

4. Melca M O Barros, Dante M Langhi Jr, José O Bordin . Warm Autoimmune hemolytic anemia: recent progress in understanding the immunobiology and the treatment. Transfus Med Rev 24(3), 195210. SS Das,Rafiq uz Zaman,Mohammed Safi. Incompatible blood transfusion: Challenging yet lifesaving in the management of acute severe autoimmune hemolytic anemia. 2014;8(2)105-108

5. Das SS, Nityanand S, Chaudhary R. Clinical and serological characterization of autoimmune hemolytic anemia in a tertiary care hospital in North India. Ann Hematol 2009;88:727-32

6. Zantek ND, Koepsell SA, Tharp DR Jr, Cohn CS. The direct antiglobulin test : a critical step in the evaluation of hemolysis. Am J Hematol. 2012 Jul; 87(7):707-9.

7. King K, Ness P. Treatment of autoimmune haemolytic anemia. Semin Hematol. 2005 42(3), 131-6.
8. Chaudhary R, Das SS, Gupta R, Khetan D. Application of flow cytometry in detection of red-cell-bound IgG in Coombs-negative AIHA. Hematology 2006 Aug; 11(4):295300. 